\title{
applied optics
}

\section{Tapered nanoscale chalcogenide fibers directly drawn from bulk glasses as optical couplers for high-index resonators}

\author{
Ozan Aktaș ${ }^{1, *}$ and Mehmet BayindiR ${ }^{1,2,3,4}$ \\ 'UNAM-National Nanotechnology Research Center, Bilkent University, Ankara, Turkey \\ ${ }^{2}$ Institute of Materials Science and Nanotechnology, Bilkent University, Ankara, Turkey \\ ${ }^{3}$ Department of Physics, Bilkent University, Ankara, Turkey \\ ${ }^{4} e$-mail: mb@4unano.com \\ *Corresponding author: aktaso@bilkent.edu.tr
}

Received 1 November 2016; revised 9 December 2016; accepted 9 December 2016; posted 12 December 2016 (Doc. ID 279955); published 11 January 2017

\begin{abstract}
We report production of air-clad tapered chalcogenide fibers by directly drawing bulk glasses between cleaved tips of tapered silica fibers. Exploiting these tapered fibers with nanoscale waists as evanescent optical couplers, we demonstrate phase-matched coupling of light into on-chip whispering gallery mode chalcogenide microresonators with coupling efficiencies as high as $\mathbf{9 5 \%}$. To the best of our knowledge, this is the first-time demonstration of critical coupling into high-index microresonators by using high-index tapered fibers. The tapered chalcogenide fibers can also be utilized as optical couplers for microresonators made of various high-index materials, as well as for nonlinear optical applications. (๑) 2017 Optical Society of America
\end{abstract}

OCIS codes: (230.5750) Resonators; (230.7370) Waveguides; (230.3990) Micro-optical devices; (060.2390) Fiber optics, infrared; (160.2750) Glass and other amorphous materials.

https://doi.org/10.1364/AO.56.000385

\section{INTRODUCTION}

In recent years, whispering gallery mode (WGM) resonators [1] have been exploited in numerous applications ranging from ultralow-threshold Raman lasers [2] and optical frequency comb generators [3] to biological sensors for single virus detection [4] because of their very efficient spatial and temporal confinement of light resulting in enhanced light-matter interactions. One of the most critical issues for practical applications of WGM resonators is yet to find a way for a robust optical coupling enabling efficient power transfer between couplers and resonators. So far, the majority of coupling schemes are based on tapered silica fibers, which have been extensively utilized for evanescent coupling of light into WGMs of microresonators not only because of their easy and low-cost production [5] but also because of their remarkable performance as the most efficient and ideal optical coupler [6]. However, tapered silica fibers are most convenient for microresonators made of materials with refractive indices similar to that of silica [7]. As an optical coupler, tapered silica fibers cannot be used to efficiently transfer optical power both to and from microresonators made of higher index $(n>1.44)$ materials because coupling efficiency is exponentially decreasing with the square of phase mismatch between propagating modes, i.e., the difference between effective refractive indices $n_{\text {eff }}$ [8]. Besides, higher order modes are more likely to be excited rather than the fundamental modes of interest as shown in chalcogenide glass (ChG) [9], germanium [10], and bismuth silicate [11] microsphere resonators. The coupling efficiency of tapered silica fibers is a lot worse for fundamental WGMs of higher index resonators with diameters $D>50 \mu \mathrm{m}$. To improve the coupling efficiency, although compromising practicality, loading of silica tapered fibers with $\mathrm{Si}$ waveguides has been proposed [12]. Other strategies have also been developed for optical coupling, some of which are high-index prism couplers for diamond sphere resonators [13], Si waveguide couplers for $\mathrm{ChG}$ sphere resonators [14], and $\mathrm{LiNbO}_{3}$ waveguide couplers for $\mathrm{LiNbO}_{3}$ disk resonators [15]. Unfortunately, these techniques still require bulky optics or pigtailed fibers at the last phase of device integration.

As an alternative, tapered fibers made of ChGs, which have unique optical properties including higher indices $(n=2.2-3.4)$, wide transparency window $(2-20 \mu \mathrm{m})$, high nonlinearity, and low two-photon absorption [16], can provide phase-matched couplers convenient for critical coupling of light into high-index WGM resonators. Tapered ChG fibers are usually drawn from step index ChG fibers, as is the case for silica fiber tapering. So far, leveraging their higher nonlinear 
refractive indices $n_{2}$ with reduced effective mode area $A_{\text {eff }}$ due to tapering [17], ChG tapered fibers have only been utilized in nonlinear optical applications, such as supercontinuum generation $[18,19]$, optical parametric oscillators [20], and photon pair generation [21].

Although tapered $\mathrm{ChG}$ fibers are promising candidates as an optical coupler for resonators made of high-index materials, they suffer from mechanical instability in air-clad tapered form with nanoscale waist diameter when tapering length is long $(>10 \mathrm{~mm})$, due to their inferior mechanical properties compared to those of silica. In addition, splicing them to silica fibers, which are often the external connectors, is very difficult and requires mechanical splices with UV-cured epoxy support. In fact, ChG fibers with polymer claddings have been fabricated and shown to be robust tapers [22,23]; however, their evanescent field is inside the cladding polymer, which has a high absorption in the mid-IR region. Tapering of a ChG core polymer cladding fiber is also vulnerable to Plateau-Rayleigh (PR) instability [24] or fragmentation during the fiber tapering process [25].

In this study, we developed a direct chalcogenide fiber tapering method, having been inspired by fabrication methods used to draw micro- and nanowires directly from bulk glasses [26,27], and demonstrated phase-matched optical coupling from a tapered $\mathrm{As}_{2} \mathrm{Se}_{3}$ fiber to WGMs of on-chip $\mathrm{As}_{2} \mathrm{Se}_{3}$ microdisk resonators with a maximum coupling efficiency of $95 \%$. The ChG microdisk WGM microresonators were fabricated by inducing PR capillary instability in a $\mathrm{ChG}\left(\mathrm{As}_{2} \mathrm{Se}_{3}\right)$ core polymer [polyethersulfone (PES)] cladding fiber [28]. The microsphere cavities formed inside the cladding were then transferred to a gold-coated substrate, where they were trimmed into disk shape. By optical characterization of the ChG microdisk WGM resonators with the tapered $\mathrm{ChG}$ fibers, we obtained quality factors reaching $Q=8.2 \times 10^{5}$ and $Q=3.0 \times 10^{5}$ in undercoupled and critically coupled regimes, respectively.

\section{FABRICATION OF TAPERED CHALCOGENIDE FIBERS}

Fabrication of ChG fiber tapers, steps for which are schematically shown in Figs. 1(a)-1(e), starts with conventional silica fiber tapering. After tapering of a SMF-28 silica fiber (core/ cladding diameter of $8.2 / 125 \mu \mathrm{m}$ ) softened in the flame of a hydrogen torch, the tapered silica fiber was cleaved from the center of the waist by scoring the surface of the fiber under tension with a sharp $\mathrm{SiC}$ blade. The diameter of the silica fiber waist was kept under $40 \mu \mathrm{m}$ to achieve a cladding guided fundamental mode. The next step was positioning of a material feeding mechanism, i.e., a thin cantilever carrying a small amount of bulk ChG $(<1 \mathrm{mg})$, between separated tips of the tapered silica fiber inside a custom-made electric heater, which was fabricated using a chromium-nickel wire with a total resistance of $1.3 \mathrm{k} \Omega$ [see Fig. 1(f)]. A picture of the setup for the transfer and tapering process of ChG is given in Fig. 1(g), showing the heater, the feeding cantilever, fiber holders on moving stages, and a long-range camera. A current of $104 \mathrm{~mA}$ through the wire of the electric heater was applied to reach temperatures $\left(>300^{\circ} \mathrm{C}\right)$ high enough to melt the $\mathrm{As}_{2} \mathrm{Se}_{3}$ glass on the cantilever. The ChG used for transfer was prepared from high-purity As and Se elements (Alfa Aesar) using a sealed-ampule melt-quenching technique [28].

Transfer of ChG to the tips of the tapered silica fiber was obtained by immersing the tips into the molten glass and then retracting them with some attached material, as shown in Figs. 2(a) and 2(b). One of the tips or both can be used in the transfer process according to the intended amount of material to be attached. After moving the feeding mechanism away, separated silica fiber tips were brought together to merge the attached material in the space between via surface tension of the glass and its surface adhesion to the silica fiber tip facets [see Figs. 2(c) and 2(d)]. At this stage just before tapering, the temperature of the system was still kept over the melting temperature of the glass. Evaporation of the glass after its splicing on both sides transforms the bottlelike shape into a uniform cylinder shape, as seen in Fig. 2(e), because the cleaved silica taper tips have fixed diameters, and evaporation occurs only on the lateral outer boundary of the glass. Tapering always starts at the weakest point between the silica tapers including the splicing points, where the diameter is the smallest, and the tensile stress is at its maximum. Further evaporation results in necking of the glass at the midpoint, which is required for symmetrical tapering. Evaporation rate can be efficiently adjusted by the temperature. Tapering process was initiated at a temperature of $325^{\circ} \mathrm{C}$ after the current of the electrical heater was set for a steady state temperature of $140^{\circ} \mathrm{C}$, which is lower than glass transition temperature $\left(T_{g}=170^{\circ} \mathrm{C}\right)$ of $\mathrm{As}_{2} \mathrm{Se}_{3}$. The tapering process took $265 \mathrm{~s}$. Because the temperature of the systems decays exponentially with a time constant, the tapering was stopped before the temperature decreased below the $T_{g}$ of the glass. (a)



(c)

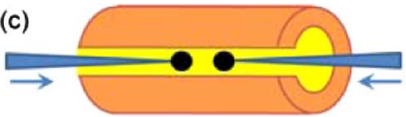

(d)

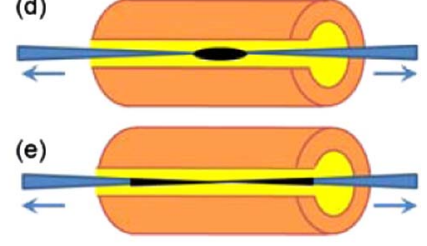

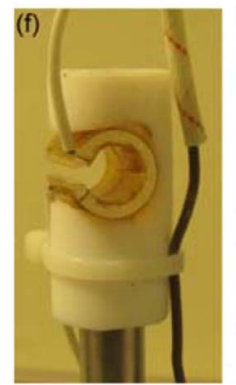

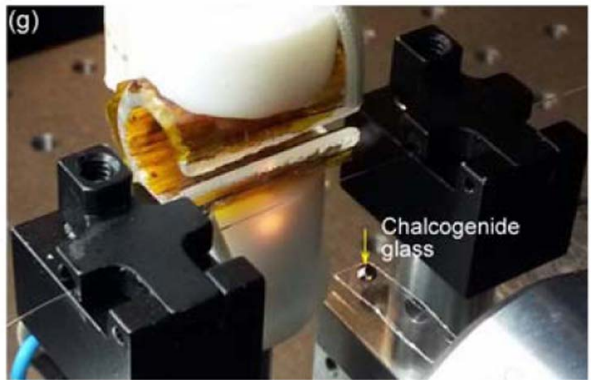

Fig. 1. Steps of ChG fiber tapering are (a) conventional silica fiber tapering and cleaving, (b) positioning of the ChG feeding mechanism, (c) transfer of some molten ChG to the tips of silica tapers, (d) merging of attached molten glasses between the silica taper tips at a temperature above the melting temperature of the glass, and (e) tapering of the ChG by separating the silica taper tips at temperatures above glass transition $T_{g}$ of the ChG. (f) Custom-made electric heater. (g) Setup used both for material transfer and direct fiber tapering of bulk glasses. 

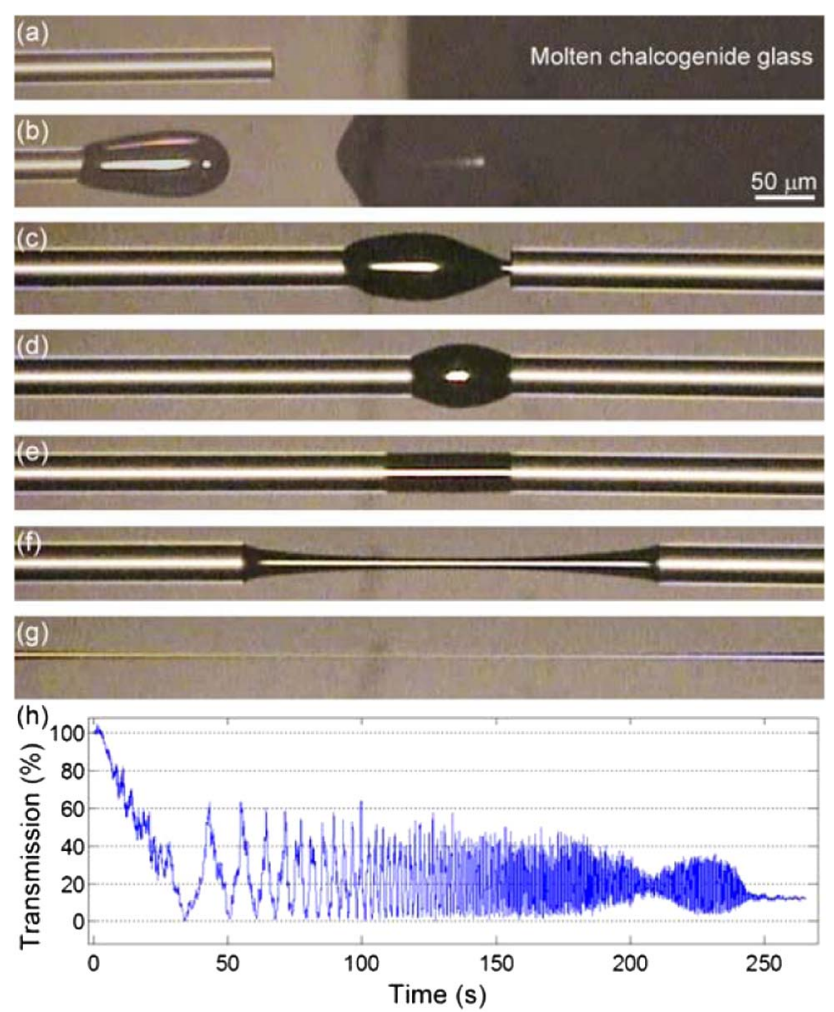

Fig. 2. Optical microscopy video snapshots of ChG transfer and fiber tapering. (a) Tapered silica fiber tip and molten bulk glass on a cantilever, and (b) attachment of some material to the tapered silica fiber tip (Visualization 1). (c) Surface adhesion of molten glass to the tapered silica fiber tips. (d) Evaporation of excess ChG material in the space between the tapered silica fiber tips. (e) Uniform cylinder of ChG between the tapered silica fiber tips. (f) Separation of the tapered silica fiber tips for ChG tapering. (g) Tapered ChG fiber between the tapered silica fiber tips (Visualization 2). (h) Optical transmission during the ChG fiber tapering.

The set temperature can be adjusted according to different tapering durations and lengths, so final temperature is always higher than $T_{g}$. As a result, a tapered ChG fiber was formed by drawing the glass bilaterally with the cleaved tips of the tapered silica fiber [see Figs. 2(f) and 2(g)], which was already connected to a laser source (Santec TLS-510) at one end and to a power meter (Newport 1935C with 918D-IR-0D3R detector) at the other end. The tapering process was monitored by a long working distance objective system (Optem Zoom 70XL) from the side opening of the heater, and the transmission of an optical input of $1 \mathrm{~mW}$ at a wavelength of $1550 \mathrm{~nm}$ was recorded during the process. A tapering loss of $9.1 \mathrm{~dB}$ was observed after an initial drop of power due to the nonadiabatic tapering. Multimode interference with a single-mode propagation regime achieved at the end, as seen in Fig. 2(h), is a common phenomenon also observed in silica fiber tapering [29].

Tapering length, which was $1.5 \mathrm{~mm}$ for this case, can be as short as $10 \mu \mathrm{m}$ or up to $10 \mathrm{~mm}$ depending on the diameter $(125 \mu \mathrm{m}>D>5 \mu \mathrm{m})$ of the tapered silica fiber tips as determined by the cleaving of the silica fiber waist. The tapered $\mathrm{ChG}$ fiber is able to remain suspended without losing its integrity under the force of gravity, demonstrating a substantial
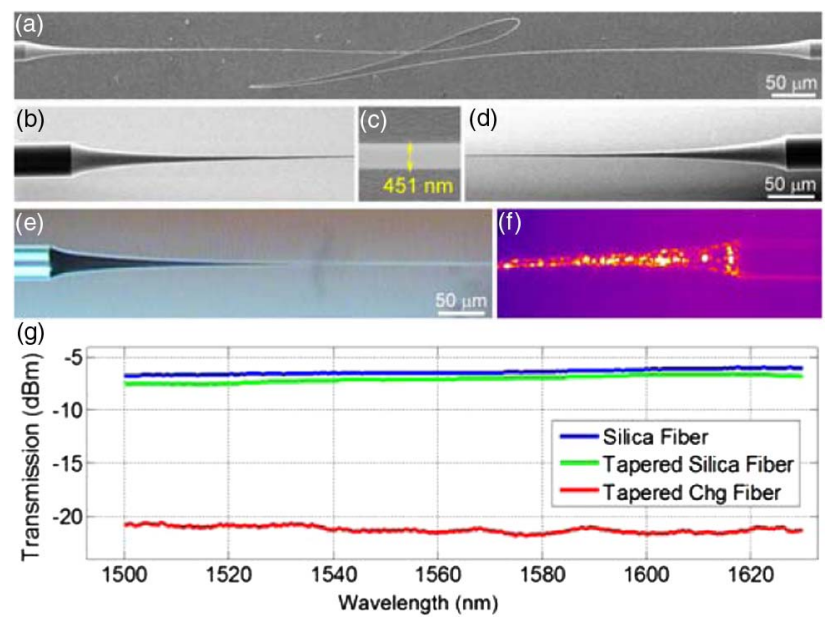

Fig. 3. SEM micrographs. (a) Tapered ChG fiber between the tips of tapered silica fibers, which were brought closer to show the whole tapered ChG fiber. (b) Left thermal splice point, (c) nanoscale diameter waist region, and (d) right thermal splice point of the tapered $\mathrm{ChG}$ fiber. (e) Optical microscopy image for contrast between ChG (black) and silica materials. (f) Thermal image showing the scattering of coupled light at the ChG taper transition region. (g) Optical transmission spectra before and after the silica tapering and after the ChG fiber tapering between the silica fiber tips.

mechanical stability. Scanning electron microscope (SEM) micrographs of the produced tapered $\mathrm{ChG}$ fiber, including waist region and left and right thermal splice points, are shown in Figs. 3(a)-3(d). The diameters of the tapered silica fiber tip and the ChG taper waist are $37 \mu \mathrm{m}$ and $451 \mathrm{~nm}$, respectively. Better contrast can be obtained at the optical microscopy image in Fig. 3(e). Thermal image showing the scattering of coupled light at the transition region is given in Fig. 3(f). A comparative result of transmissions before and after the silica fiber tapering and after the $\mathrm{ChG}$ fiber tapering between the tapered silica fiber tips is given in Fig. 3(g).

We obtained a broadband transmission for the tapered ChG fiber, which was drawn until we reached single-mode regime at a nanoscale waist diameter. The total insertion loss was measured to be $21.1 \mathrm{~dB}$, which is the sum of $6.4 \mathrm{~dB}$ loss that already existed before the silica fiber tapering process and 14.7 $\mathrm{dB}$ loss created afterward. The initial loss consists of $3.4 \mathrm{~dB}$ loss due to silica-to-silica mechanical coupling on both sides of the tapered silica fiber and $3 \mathrm{~dB}$ loss due to a fiber coupler used for $50 \%$ splitting of the output power, which can be effectively mitigated by thermal splicing of the silica fibers, and direct reading of the output power. The subsequent loss after the process consists of $0.6 \mathrm{~dB}$ silica fiber tapering loss, $1 \mathrm{~dB}$ Fresnel loss for both ChG/silica interface, 9.1 dB ChG fiber tapering loss, and $4 \mathrm{~dB}$ loss due to nonperfect cleaving of the tapered silica fiber and mode mismatch. A loss under $0.1 \mathrm{~dB}$ can be obtained for silica fiber tapering using the flamebrushing method. As achieved recently, ChG fiber tapering loss can be reduced to at least $5 \mathrm{~dB}$ by tapering a longer cylinder of ChG initially spliced to the silica tapers, improving the adiabaticity. Starting ChG fiber tapering with smaller diameter silica tips $(D<20 \mu \mathrm{m})$ is also more favorable for adiabatic 
transition of the cladding guided modes, reducing the loss of mode mismatch. In addition to nonadiabaticity, ChG fiber tapering loss is increased by surface scattering and Rayleigh scattering due to density fluctuations caused by different evaporating rates of As and Se, changing the stoichiometry of the glass. Repetitive thermal annealing of the same bulk glass after multiple material transfers was observed to adversely affect the tapering process because of the crystallization started in the material. In principle, angle cleaved silica fiber tips can eliminate the Fresnel losses, and unlike our tapering process, which occurred under ambient conditions, tapering under inert gas atmosphere can reduce oxidation effects degrading ChGs.

\section{FABRICATION OF CHALCOGENIDE RESONATORS}

For the exploitation of the tapered ChG fibers as evanescent couplers, we produced microdisk cavity WGM resonators made of $\mathrm{As}_{2} \mathrm{Se}_{3}$ glass $(n=2.7)$, as shown in Figs. 4(a)-4(d), extending what we developed in a previous study [28]. Starting with an $\mathrm{As}_{2} \mathrm{Se}_{3}$ core $(\varnothing 34 \mu \mathrm{m})$ PES cladding $(\varnothing 170 \mu \mathrm{m})$ fiber, we induced formation of microspheres in the polymer cladding at
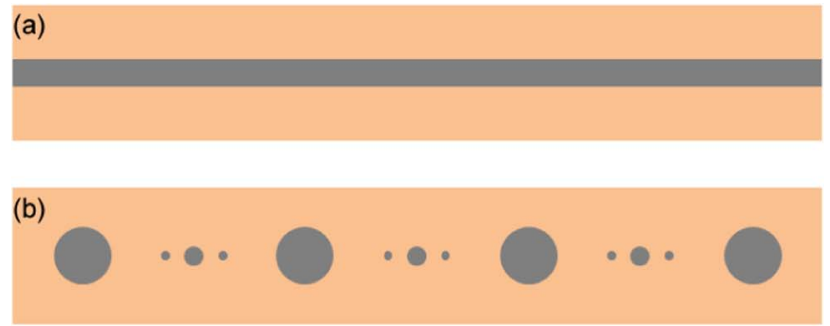

(c)
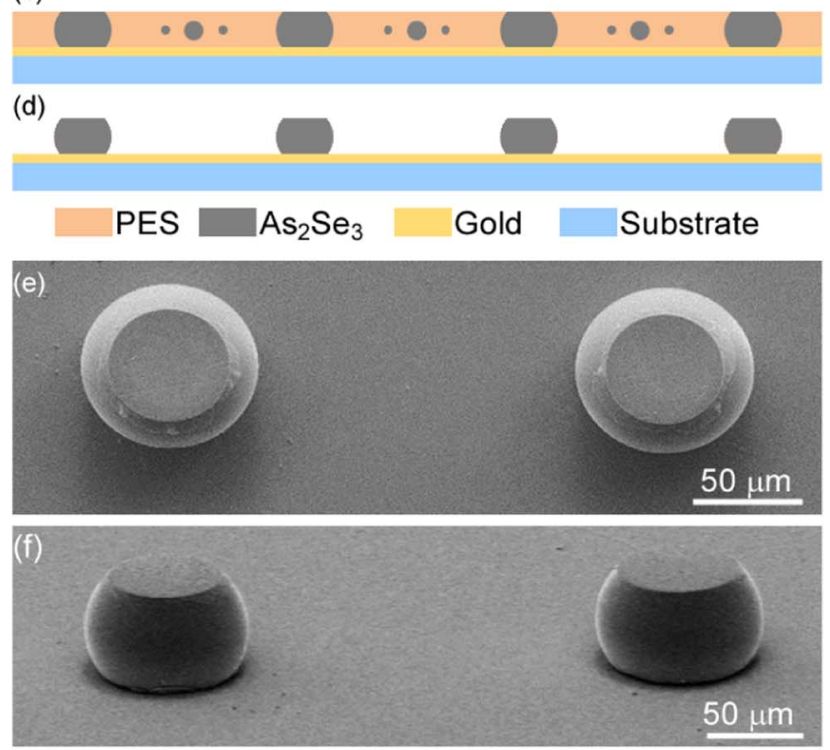

Fig. 4. Production and integration of microdisk cavity ChG resonators. (a) $\mathrm{As}_{2} \mathrm{Se}_{3}$ core and PES cladding fiber. (b) In-fiber microsphere formation by inducing PR instability. (c) Attachment of the bottom-trimmed fiber containing ordered microcavities on a goldcoated substrate, and top-side abrasion of the fiber by sandpapering. (d) Dissolution of the polymer encapsulation by organic solvents. SEM micrograph of on-chip ChG microdisk WGM resonators from (e) top and (f) oblique perspectives. a temperature of $300^{\circ} \mathrm{C}$ by PR capillary instability. Later, the bottom side of the fiber was rubbed away to uncover contact surfaces for the embedded microspheres by using sheets of $\mathrm{SiC}$ sandpaper with a size of abrasive particles decreasing from $5 \mu \mathrm{m}$ to $1 \mu \mathrm{m}$. To promote adhesion after transfer of the bottom-trimmed fiber onto a gold-coated substrate, we applied heat to increase temperature up to $240^{\circ} \mathrm{C}$, which is higher than both glass transition temperature of $\mathrm{As}_{2} \mathrm{Se}_{3}\left(T_{g}=170^{\circ} \mathrm{C}\right)$ glass and PES $\left(T_{g}=210^{\circ} \mathrm{C}\right)$ polymer. In addition to these steps, we trimmed the top side of the fiber to transform cavities into a disk shape with suppressed higher order polar modes. At the last step, which was the dissolution of the polymer encapsulation by organic solvents (dichloromethane) and removal of all satellite microspheres, we obtained on-chip ChG microdisk cavity resonators as seen in the SEM micrographs of Figs. 4(e) and 4(f). Average diameter of the $\mathrm{ChG}$ microresonators is $68 \pm 0.5 \mu \mathrm{m}$.

\section{MEASUREMENT}

One of the on-chip ChG microdisk WGM resonators was positioned with a three-axis closed-loop piezo stage (NanoMax-TS) close to a tapered $\mathrm{ChG}$ fiber assuring alignment with the equator of the ChG microresonator as shown in Figs. 5(a) and 5(b). The tension of the tapered ChG fiber was adjusted to counter attraction between the fiber and the ChG resonator, due to electrostatic and van der Waals forces. Furthermore, we moved
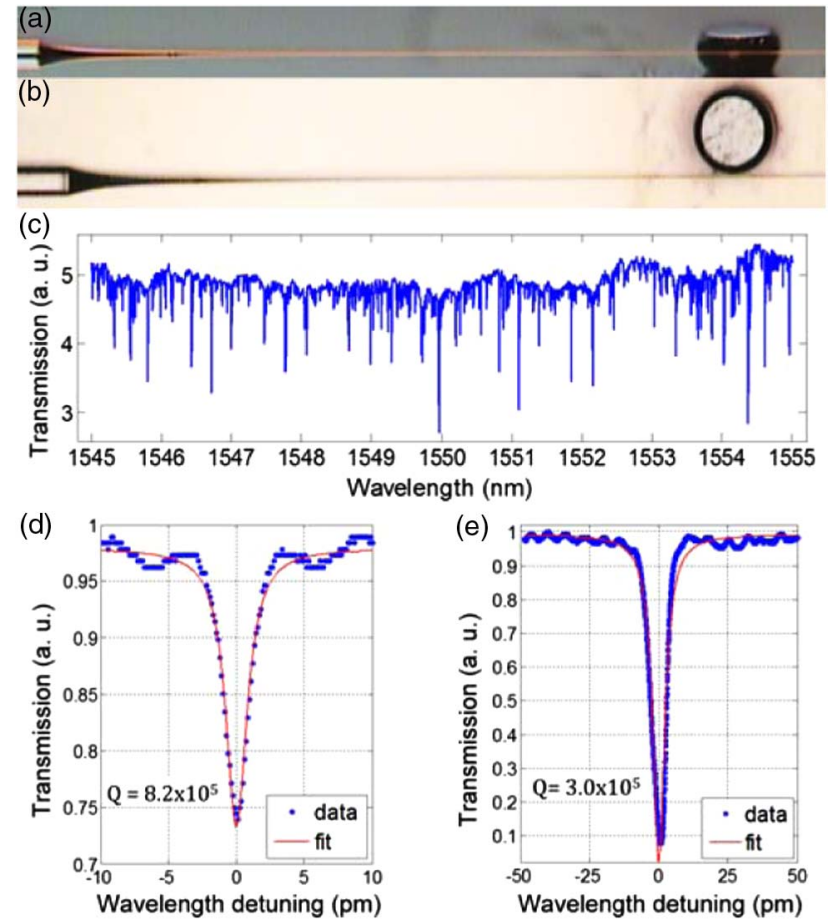

Fig. 5. Optical characterizations of the ChG microdisk WGM resonators with the tapered ChG fibers. Optical microscopy images from (a) side and (b) top perspectives, showing a tapered ChG fiber coupled to a ChG microdisk WGM resonator. The tapered silica fiber tip thermally spliced to the tapered ChG fiber can also be seen in the images. (c) Wide-range optical transmission spectrum of the ChG microresonator showing two periodic families of WGM modes. (d) Optical transmission spectra of the resonant mode around the wavelength of $1551 \mathrm{~nm}$ in the (d) undercoupled and (e) critically coupled regimes. 
the $\mathrm{ChG}$ microresonator along the tapered $\mathrm{ChG}$ fiber until we found the phase-matching diameter, and polarization was adjusted for optimum light coupling. The laser wavelength of the $\mathrm{CW}$ source was scanned continuously for a $10 \mathrm{~nm}$ wavelength range with a scan rate of $10 \mathrm{~nm} / \mathrm{s}$. We measured the wide-range transmission spectrum as given in Fig. 5(c). After setting the wavelength scan rate to $1 \mathrm{~nm} / \mathrm{s}$ and scan range to $50 \mathrm{pm}$ with the central wavelength at $1551 \mathrm{~nm}$ for a clear resonant mode, we increased the separation between the tapered fiber and the ChG resonator to measure transmission in the undercoupled regime [see Fig. 5(d)]. We then moved the ChG microresonator gradually closer to the tapered ChG fiber to reach the critical coupling regime, which is the $13 \mathrm{~dB}$ drop of optical transmission, as shown in Fig. 5(e). Lorentzian fits to the resonance mode in the transmission spectra show a quality factor of $Q=8.2 \times 10^{5}$ in the undercoupled regime and a quality factor of $Q=3 \times 10^{5}$ in the critically coupled regime. It is critical to use input powers lower than $1 \mu \mathrm{W}$ to eliminate thermo-optic and nonlinear effects observed in ChG microresonators, which can distort the Lorentzian shape of the resonant modes.

\section{RESULTS AND DISCUSSION}

As a result of optical characterization of a tapered $\mathrm{As}_{2} \mathrm{Se}_{3} \mathrm{ChG}$ fiber coupled with WGMs of the $\mathrm{As}_{2} \mathrm{Se}_{3}$ microdisk resonators of $D=68 \mu \mathrm{m}$, we obtained a maximum coupling efficiency of $13 \mathrm{~dB}(95 \%)$ and a maximum quality factor of $Q=8.2 \times 10^{5}$, which is close to the intrinsic quality factor in the undercoupled regime. We previously obtained a maximum coupling efficiency of $8.7 \mathrm{~dB}$ and an intrinsic quality factor of $Q=7.9 \times 10^{5}$ for coupling with WGMs of $\mathrm{As}_{2} \mathrm{Se}_{3}$ microsphere resonators of $D=50 \mu \mathrm{m}$ with tapered silica fibers [28]. Although $Q$ factors obtained in this study are comparable (slightly higher) to those previously obtained with silica tapered fibers, there is a remarkable increase of $4.3 \mathrm{~dB}$ in the maximum optical coupling efficiency observed for WGMs of the ChG microresonators with tapered ChG fibers. Maximum coupling efficiencies, which were obtained by other groups, are $10 \mathrm{~dB}$ for a silica tapered fiber coupled with $\mathrm{As}_{2} \mathrm{Se}_{3}$ microspheres of $D=9.2 \mu \mathrm{m}$ [9], and $7 \mathrm{~dB}$ for silicon waveguide couplers coupled with $\mathrm{As}_{2} \mathrm{Se}_{3}$ microspheres of $D>55 \mu \mathrm{m}$ [14]. We expect similar improvements in coupling efficiencies that are to be obtained for tapered ChG fibers coupled with other resonators made of high-index materials, such as $\mathrm{Ge}, \mathrm{Si}$, and $\mathrm{LiNbO}_{3}$.

Fundamental WGMs are of interest for sensing or nonlinear optical applications, because they possess not only high-quality factors but also the smallest mode volume. Achieving critical optical coupling with the fundamental WGMs, which is the $100 \%$ power transfer both to and from a resonator, is therefore highly desired. However, this is only favorable with phasematched couplers for high-index $(n>2)$ microresonators having diameters greater than $50 \mu \mathrm{m}$. Perfect phase matching for the fundamental WGMs can only be fulfilled by tapered fibers with equal or greater material index than effective indices of the WGMs in a microresonator. ChG tapered fibers with refractive index $n>2.2$ will be a good match for most of the high-index resonators as efficient optical couplers. Moreover, starting with extremely small quantities of ChG instead of fibers, thermal splicing to silica fibers in the beginning of a tapering process and ease of tapering with electric heaters at low temperatures will be of importance for practical applications of WGM microresonators.

In this work, we also demonstrated that chalcogenide nanofibers can be obtained on the tip of tapered silica fibers. Because ChGs have large material dispersion, zero total dispersion occurs at nanoscale dimensions, so with this technique any nanoscale diameter is achievable to engineer total dispersion during tapering. Besides, the absence of long nontapered ChG fiber parts, which have high normal dispersion, can be of great advantage in terms of pre-chirp compensation required in ultrafast optical applications.

Because tapering is a thermal process based on electrical heating, the surface of the ChG nanofibers is very smooth. In addition, we observed superior mechanical endurance for the Chg nanofibers, which can be elastically transformed into loops by bringing the tapered silica fibers closer, without any mechanical and optical degradation. Being thermally spliced to silica fibers, nanofibers could be an important step for achieving in-line novel and compact fiber functional devices.

\section{CONCLUSIONS}

In conclusion, we demonstrated production of air-clad nanoscale waist diameter tapered ChG fibers directly drawn from bulk glasses of $\mathrm{As}_{2} \mathrm{Se}_{3}$ with extremely low starting quantities inside an electric heater. This technique can also be applied to other commonly used ChG glasses, such as $\mathrm{As}_{2} \mathrm{~S}_{3}$, Ge$\mathrm{Sb}-\mathrm{Te}$, and Ge-As-Se-Te. The tapered ChG fibers are thermally spliced to silica fibers on both sides, eliminating the need for a ChG fiber in the first place and postprocess splicing or mechanical coupling. Furthermore, noncircular tapered ChG fibers can be produced if the silica taper tips have noncircular shapes, which can be used to induce birefringence into the fibers. As an application, we demonstrated phase-matched coupling of light into on-chip $\mathrm{As}_{2} \mathrm{Se}_{3}$ microdisk cavity WGM resonators with a maximum coupling efficiency of $95 \%$ and a maximum quality factor of $Q=8.2 \times 10^{5}$, verifying optical quality of our $\mathrm{ChG}$ microresonators and enhanced optical coupling with our tapered ChG fibers. To the best of our knowledge, this is the first time for demonstration of optical coupling into high-index WGM resonators with high-index tapered fibers. These tapered $\mathrm{ChG}$ fibers can be exploited as evanescent optical couplers for other resonators made of high-index amorphous glasses and high-index crystalline materials, such as $\mathrm{CaF}_{2}, \mathrm{Ge}, \mathrm{Si}$, and $\mathrm{LiNbO}_{3}$. Tapered $\mathrm{ChG}$ fibers can also be drawn between cleaved tips of other fibers made of hightemperature materials, such as $\mathrm{ZnSe}, \mathrm{Si}$, and $\mathrm{Ge}$. In addition, owing to wide-range optical transmission of ChGs, air-clad tapered ChG fibers are very convenient for mid-IR applications. In particular, the combinations of intrinsic high nonlinearity of ChGs, the reduced effective mode area $A_{\text {eff }}$ due to nanoscale diameters, and the absence of nontapered fiber parts can be leveraged in nonlinear optical applications, such as nonlinear label-free biosensing [30], third harmonic generation [31], and generation of entangled photon triplets [32], where the promise of tapered $\mathrm{ChG}$ fibers is highlighted. 
Funding. European Research Council (ERC) (307357).

Acknowledgment. We thank Dr. Erol Özgür for his help in paper editing and his valuable suggestions.

\section{REFERENCES}

1. K. J. Vahala, "Optical microcavities," Nature 424, 839-846 (2003).

2. S. M. Spillane, T. J. Kippenberg, and K. J. Vahala, "Ultralow threshold Raman laser using a spherical dielectric microcavity," Nature 415, 621-623 (2002).

3. T. J. Kippenberg, R. Holzwarth, and S. A. Diddams, "Microresonatorbased optical frequency combs," Science 332, 555-559 (2011).

4. F. Vollmer, S. Arnold, and D. Keng, "Single virus detection from the reactive shift of a whispering-gallery mode," Proc. Natl. Acad. Sci. USA 105, 20701-20704 (2008).

5. S. W. Harun, K. S. Lim, C. K. Tio, K. Dimyati, and H. Ahmad, "Theoretical analysis and fabrication of tapered fiber," Optik 124, 538-543 (2013).

6. S. M. Spillane, T. J. Kippenberg, O. J. Painter, and K. J. Vahala, "Ideality in a fiber-taper-coupled microresonator system for application to cavity quantum electrodynamics," Phys. Rev. Lett. 91, 043902 (2003).

7. J. C. Knight, G. Cheung, F. Jacques, and T. A. Birks, "Phase-matched excitation of whispering-gallery-mode resonances by a fiber taper," Opt. Lett. 22, 1129-1131 (1997).

8. B. E. Little, J.-P. Laine, and H. A. Haus, "Analytic theory of coupling from tapered fibers and half-blocks into microsphere resonators," J. Lightwave Technol. 17, 704-715 (1999).

9. C. Grillet, S. N. Bian, E. C. Magi, and B. J. Eggleton, "Fiber taper coupling to chalcogenide microsphere modes," Appl. Phys. Lett. 92, 171109 (2008).

10. P. Wang, T. Lee, M. Ding, A. Dhar, T. Hawkins, P. Foy, Y. Semenova, Q. Wu, J. Sahu, G. Farrell, J. Ballato, and G. Brambilla, "Germanium microsphere high-Q resonator," Opt. Lett. 37, 728-730 (2012).

11. P. Wang, G. S. Murugan, T. Lee, M. Ding, G. Brambilla, Y. Semenova, Q. Wu, F. Koizumi, and G. Farrell, "High-Q bismuth-silicate nonlinear glass microsphere resonators," IEEE Photon. J. 4, 1013-1020 (2012).

12. P. Latawiec, M. J. Burek, V. Venkataraman, and M. Lončar, "Waveguide-loaded silica fibers for coupling to high-index microresonators," Appl. Phys. Lett. 108, 031103 (2016).

13. V. S. Ilchenko, A. M. Bennett, P. Santini, A. A. Savchenkov, A. B. Matsko, and L. Maleki, "Whispering gallery mode diamond resonator," Opt. Lett. 38, 4320-4323 (2013).

14. D. H. Broaddus, M. A. Foster, I. H. Agha, J. T. Robinson, M. Lipson, and A. L. Gaeta, "Silicon-waveguide-coupled high-Q chalcogenide microspheres," Opt. Express 17, 5998-6003 (2009).

15. G. N. Conti, S. Berneschi, F. Cosi, S. Pelli, S. Soria, G. C. Righini, M. Dispenza, and A. Secchi, "Planar coupling to high- $Q$ lithium niobate disk resonators," Opt. Express 19, 3651-3656 (2011).

16. B. J. Eggleton, B. Luther-Davies, and K. Richardson, "Chalcogenide photonics," Nat. Photonics 5, 141-148 (2011).

17. E. C. Mägi, L. B. Fu, H. C. Nguyen, M. R. E. Lamont, D. I. Yeom, and B. J. Eggleton, "Enhanced Kerr nonlinearity in sub-wavelength diameter $\mathrm{As}_{2} \mathrm{Se}_{3}$ chalcogenide fiber tapers," Opt. Express 15 10324-10329 (2007).

18. D. D. Hudson, S. A. Dekker, E. C. Mägi, A. C. Judge, S. D. Jackson, E. Li, J. S. Sanghera, L. B. Shaw, I. D. Aggarwal, and B. J. Eggleton, "Octave spanning supercontinuum in an $\mathrm{As}_{2} \mathrm{~S}_{3}$ taper using ultralow pump pulse energy," Opt. Lett. 36, 1122-1124 (2011).

19. C. W. Rudy, A. Marandi, K. L. Vodopyanov, and R. L. Byer, "Octave spanning supercontinuum generation in in situ tapered $\mathrm{As}_{2} \mathrm{~S}_{3}$ fiber pumped by a thulium-doped fiber laser," Opt. Lett. 38, 2865-2868 (2013).

20. N. Abdukerim, L. Li, and M. Rochette, "Chalcogenide-based optical parametric oscillator at $2 \mu \mathrm{m}$," Opt. Lett. 41, 4364-4367 (2016).

21. E. Meyer-Scott, A. Dot, R. Ahmad, L. Li, M. Rochette, and T. Jennewein, "Power-efficient production of photon pairs in a tapered chalcogenide microwire," Appl. Phys. Lett. 106, 081111 (2015).

22. S. Shabahang, G. Tao, J. J. Kaufman, and A. F. Abouraddy, "Dispersion characterization of chalcogenide bulk glass, composite fibers, and robust nanotapers," J. Opt. Soc. Am. B 30, 2498-2506 (2013).

23. C. Baker and M. Rochette, "High nonlinearity and single-mode transmission in tapered multimode $\mathrm{As}_{2} \mathrm{Se}_{3}$-PMMA fibers," IEEE Photon. J. 4, 960-969 (2012).

24. S. Shabahang, J. J. Kaufman, D. S. Deng, and A. F. Abouraddy, "Observation of the Plateau-Rayleigh capillary instability in multimaterial optical fibers," Appl. Phys. Lett. 99, 161909 (2011).

25. S. Shabahang, G. Tao, J. J. Kaufman, Y. Qiao, L. Wei, T. Bouchenot, A. P. Gordon, Y. Fink, Y. Bai, R. S. Hoy, and A. F. Abouraddy, "Controlled fragmentation of multimaterial fibres and films via polymer cold-drawing," Nature 534, 529-533 (2016).

26. L. Tong, L. Hu, J. Zhang, J. Qu, Q. Yang, J. Lou, Y. Shen, J. He, and Z. Ye, "Photonic nanowires directly drawn from bulk glasses," Opt. Express 14, 82-87 (2006).

27. N.-K. Chen, P.-W. Kuan, J. Zhang, L. Zhang, L. Hu, C. Lin, and L. Tong, "Multicolor upconversion emissions in $\mathrm{Tm}^{3+} / \mathrm{Er}^{3+}$ codoped tellurite photonic microwire between silica fiber tapers," Opt. Express 18, 25615-25626 (2010).

28. O. Aktas, E. Ozgur, O. Tobail, M. Kanik, E. Huseyinoglu, and M. Bayindir, "A new route for fabricating on-chip chalcogenide microcavity resonator arrays," Adv. Opt. Mater. 2, 618-625 (2014).

29. F. Orucevic, V. Lefèvre-Seguin, and J. Hare, "Transmittance and near-field characterization of sub-wavelength tapered optical fibers," Opt. Express 15, 13624-13629 (2007).

30. C. Markos and O. Bang, "Nonlinear label-free biosensing with high sensitivity using $\mathrm{As}_{2} \mathrm{~S}_{3}$ chalcogenide tapered fiber," J. Lightwave Technol. 33, 2892-2898 (2015).

31. A. Coillet and P. Grelu, "Third-harmonic generation in optical microfibers: From silica experiments to highly nonlinear glass prospects," Opt. Commun. 285, 3493-3497 (2012).

32. M. Corona, K. Garay-Palmett, and A. B. U'Ren, "Experimental proposal for the generation of entangled photon triplets by third-order spontaneous parametric downconversion in optical fibers," Opt. Lett. 36, 190-192 (2011). 